\title{
CONDITIONAL FULL SUPPORT OF GAUSSIAN PROCESSES WITH STATIONARY INCREMENTS
}

\author{
DARIO GASBARRA,* University of Helsinki \\ TOMMI SOTTINEN, ${ }^{* *}$ University of Vaasa \\ HARRY VAN ZANTEN, ${ }^{* * *}$ Eindhoven University of Technology
}

\begin{abstract}
We investigate the conditional full support (CFS) property, introduced in Guasoni et al. (2008a), for Gaussian processes with stationary increments. We give integrability conditions on the spectral measure of such a process which ensure that the process has CFS or not. In particular, the general results imply that, for a process with spectral density $f$ such that $f(\lambda) \sim c_{1} \lambda^{p} \mathrm{e}^{-c_{2} \lambda^{q}}$ for $\lambda \rightarrow \infty$ (with necessarily $p<1$ if $q=0$ ), the CFS property holds if and only if $q<1$.
\end{abstract}

Keywords: Conditional full support; conditional law; Gaussian process; processes with stationary increments; spectral measure

2010 Mathematics Subject Classification: Primary 60G15

Secondary $60 \mathrm{G} 30$

\section{Introduction}

In mathematical finance there has lately been considerable interest in pushing beyond the by now classical setting of asset prices modelled by semimartingales; cf. e.g. Delbaen and Schachermayer (2006) and the references therein. New approaches that either restrict the class of allowed trading strategies or introduce transaction costs allow, e.g. for the use of fractional Brownian motion to model (log) prices; cf. Bender et al. (2008) Guasoni et al. (2008a), and Jarrow et al. (2009). The results of Guasoni et al. (2008a), (2008b) show that when transaction costs are introduced, a satisfactory arbitrage theory can be built up using continuous price processes that only satisfy a mild condition on their conditional support (we will give a precise definition below).

To understand the scope of the newly developed theories, it has become important to investigate the conditional support properties for classes of stochastic processes. Guasoni et al. (2008a) proved that the fractional Brownian motion with arbitrary Hurst parameter has the desired property. This was recently generalized in Cherny (2008), who proved the conditional full support (CFS) property for a class of Gaussian processes that can be written as a moving average with respect to ordinary Brownian motion.

In the present paper we focus on the class of continuous, centered Gaussian processes $X$ with stationary increments, starting at 0 , i.e. $X_{0}=0$. We will briefly call such a process a continuous

\footnotetext{
Received 13 July 2009; revision received 17 February 2011.

* Current address: Department of Mathematics and Statistics, University of Jyväskylä, PO Box 35 (MaD), FIN-40014, Jyväskylä, Finland. Email address: dario.d.gasbarra@jyu.fi

** Postal address: Department of Mathematics and Statistics, University of Vaasa, PO Box 700, FIN-65101, Vaasa, Finland. Email address: tommi.sottinen@uwasa.fi

*** Postal address: Department of Mathematics, Eindhoven University of Technology, PO Box 513, 5600 MB Eindhoven, The Netherlands. Email address: j.h.v.zanten@tue.nl
} 
Gaussian si-process. It is a classical fact that with each such process we can associate a spectral measure. This is a symmetric Borel measure $\mu$ on the line such that

$$
\mathrm{E} X_{s} X_{t}=\int_{\mathbb{R}} \frac{\left(\mathrm{e}^{\mathrm{i} \lambda s}-1\right)\left(\mathrm{e}^{-\mathrm{i} \lambda t}-1\right)}{\lambda^{2}} \mu(\mathrm{d} \lambda)
$$

for all $s, t \geq 0$ (cf. Doob (1953)). If $\mu$ admits a Lebesgue density, we call this the spectral density of the process. The prime example is the fractional Brownian motion with Hurst index $H \in(0,1)$, which has spectral density

$$
f(\lambda)=\frac{\sin (\pi H) \Gamma(1+2 H)}{2 \pi}|\lambda|^{1-2 H} \mathrm{~d} \lambda ;
$$

see, e.g. Samorodnitsky and Taqqu (1994, pp. 318-325). The continuous moving average processes considered in Cherny (2008) are particular examples of continuous Gaussian siprocesses. Included in this class are the continuous Gaussian si-processes with spectral densities of the form

$$
f(\lambda)=\lambda^{2}|\hat{K}(\lambda)|^{2}
$$

where $\hat{K}$ is the Fourier transform of a function $K \in L^{2}[0, \infty)$. We use this connection in the proof of our main theorem.

The CFS property of a process indexed by a time interval $[0, T]$ roughly means that, given the information up to some time $t \in[0, T]$, the process can go anywhere after time $t$ with positive probability. To give the precise definition, let $X=\left(X_{t}: t \in[0, T]\right)$ be a continuous stochastic process defined on a probability space $(\Omega, \mathcal{F}, \mathrm{P})$. Let $\left(\mathcal{F}_{t}\right)$ be its natural filtration. The process is said to have CFS if, for all $t \in[0, T]$, the conditional law of $\left(X_{u}: u \in[t, T]\right)$ given $\mathcal{F}_{t}$ almost surely has support $C_{X_{t}}[t, T]$, where $C_{x}[t, T]$ is the space of continuous functions $f$ on $[t, T]$ satisfying $f(t)=x$. Equivalently, this means that, for all $t \in[0, T], f \in C_{0}[t, T]$, and $\varepsilon>0$,

$$
\mathrm{P}\left(\sup _{u \in[t, T]}\left|X_{u}-X_{t}-f(u)\right|<\varepsilon \mid \mathcal{F}_{t}\right)>0
$$

almost surely. According to Lemma 2.9 of Guasoni et al. (2008a), the CFS condition is in fact equivalent to the seemingly stronger condition that is obtained by replacing the deterministic times $t$ in the definition by stopping times.

The goal of this paper is to give spectral conditions for a Gaussian si-process to have CFS. It turns out that we can give rather precise conditions on the tails of the spectral density. The results quantify the intuitively reasonable statement that a Gaussian si-process has CFS if and only if the tails of its spectral measure are heavy enough. The sufficient tail condition for CFS is very mild and easy to verify in concrete cases, thus providing a wide class of processes potentially suitable for asset price modelling in the context of Guasoni et al. (2008a), (2008b).

\section{Results}

Our main result gives integrability conditions on the spectral measure of a Gaussian siprocess which ensure that the process has the CFS property or not.

Being a symmetric Borel measure on the line, the spectral measure $\mu$ of a Gaussian si-process can always be written as $\mu(\mathrm{d} \lambda)=f(\lambda) \mathrm{d} \lambda+\mu_{s}(\mathrm{~d} \lambda)$ with $f$ a nonnegative, symmetric, Borel measurable function and $\mu_{s}$ a symmetric Borel measure orthogonal to the Lebesgue measure. As usual, we call $f$ the density of the absolutely continuous part of $\mu$. 
Theorem 2.1. Let $X=\left(X_{t}: t \in[0, T]\right)$ be a continuous Gaussian si-process with spectral measure $\mu$. Let $f$ be the density of the absolutely continuous part of $\mu$.

(i) If, for some $\lambda_{0}>0$,

$$
\int_{\lambda_{0}}^{\infty} \frac{\log f(\lambda)}{\lambda^{2}} \mathrm{~d} \lambda>-\infty
$$

then $X$ has CFS.

(ii) If, for some $a, \lambda_{0}>0$,

$$
\int_{\lambda_{0}}^{\infty} \mathrm{e}^{a \lambda} \mu(\mathrm{d} \lambda)<\infty
$$

then $X$ does not have CFS.

To illustrate the sharpness of this result, suppose for instance that, for $c_{1}, c_{2}>0, p \in \mathbb{R}$, and $q \geq 0$, the spectral density $f$ of the continuous Gaussian si-process $X$ satisfies

$$
f(\lambda) \sim c_{1} \lambda^{p} \mathrm{e}^{-c_{2} \lambda^{q}}
$$

as $\lambda \rightarrow \infty$ (with necessarily $p<1$ if $q=0$ ). Then by combining parts (i) and (ii) of Theorem 2.1, we see that the CFS property holds if and only if $q<1$. Taking $q=0$ and $p=1-2 H$ for $H \in(0,1)$, this confirms the result of Guasoni et al. (2008a) which states that the fractional Brownian motion with Hurst parameter $H \in(0,1)$ has CFS. In fact, any Gaussian si-process with a spectral density with a power tail has CFS. Another concrete example is the integrated Ornstein-Uhlenbeck process, which corresponds to $q=0$ and $p=-2$.

Processes with $q \in(0,1)$ are in some sense peculiar. On the one hand, they are 'very predictable' in the sense that their sample paths are infinitely often differentiable. Indeed, since the spectral measure has a finite second moment in this case, the si-process can be written as the integral of a stationary Gaussian process with the same spectral measure (cf. Doob (1953, p. 558)). Since the spectral measure has in fact finite moments of all orders if $q>0$, the latter stationary process has derivatives of all orders; see again Doob (1953, Section XI.9). On the other hand, for $q \in(0,1)$, the Gaussian si-process with spectral density (2.1) does have CFS. So in the context of Guasoni et al. (2008a), (2008b), modelling log prices with such a smooth process does not introduce arbitrage opportunities. This is of course completely different from what happens in classical theory.

The proof of Theorem 2.1 employs Theorem 1.1 of Cherny (2008) on Brownian moving averages: the moving average process

$$
X_{t}=\int_{-\infty}^{t}(K(t-s)-K(-s)) \mathrm{d} B_{s},
$$

where $B$ is a standard Brownian motion, and $K$ vanishes on the negative half-line and satisfies

$$
\int_{\mathbb{R}}(K(t-s)-K(-s))^{2} \mathrm{~d} s<\infty
$$

for all $t \geq 0$, has CFS. Conversely, our Theorem 2.1 also implies CFS for some moving averages.

Corollary 2.1. A continuous Brownian moving average process $X=\left(X_{t}: t \in[0, T]\right)$ as in (2.2), with $K \in L^{2}[0, \infty)$ a nontrivial kernel, has $C F S$. 
It should be noted that Theorem 1.1 of Cherny (2008) is more general than Corollary 2.1. Cherny considered nontrivial kernels $K$ that satisfy the less restrictive condition that $\int(K(t-$ $s)-K(-s))^{2} \mathrm{~d} s<\infty$ for all $t \geq 0$. Theorem 2.1(i) and Cherny's result should be seen as complementing each other. Some processes have tractable spectral measures, or are in fact defined through their spectrum, while a moving average representation might not exist or is untractable. In such cases Theorem 2.1 can be used to investigate the CFS property. Cherny's result is appropriate for situations where a moving average representation is known to exist, while the spectral measure may be untractable.

\section{Proof of the main result}

\subsection{Auxiliary lemmas}

The two lemmas presented in this subsection are used in the proof of Theorem 2.1, but are also useful in their own right. They state that the CFS property is preserved by an equivalent change of measure and by adding an independent continuous process.

Lemma 3.1. Let $X=\left(X_{t}: t \in[0, T]\right)$ and $Y=\left(Y_{t}: t \in[0, T]\right)$ be two continuous processes. If the laws of $X$ and $Y$ are equivalent, then $X$ has $C F S$ if and only if $Y$ has CFS.

Proof. Consider the canonical space $(\Omega, \mathcal{F})$ of continuous functions on $[0, T]$. On $(\Omega, \mathcal{F})$, let $\mathrm{P}_{X}$ and $\mathrm{P}_{Y}$ be the distributions of the processes $X$ and $Y$, respectively. It suffices to show that, for every sub- $\sigma$-field $g \subseteq \mathcal{F}$ and $A \in \mathcal{F}$, it holds that $\mathrm{P}_{X}(A \mid g)>0, \mathrm{P}_{X}$-almost surely if and only if $\mathrm{P}_{Y}(A \mid g)>0, \mathrm{P}_{Y}$-almost surely. This is straightforward to verify and left to the reader.

The next lemma states that, for independent continuous processes $X$ and $Y$, the process $X+Y$ inherits the CFS property from $X$. This is a consequence of the fact that, conditionally on $Y$, the process $X+Y$ is a CFS process offset by a continuous function (the path $Y$ ). Hence, conditionally on $Y$, the process $X+Y$ has CFS. By standard properties of conditional expectations, this is then true unconditionally as well.

Lemma 3.2. Let $X=\left(X_{t}: t \in[0, T]\right)$ and $Y=\left(Y_{t}: t \in[0, T]\right)$ be two independent continuous processes. If $X$ has CFS then $X+Y$ has CFS as well.

Proof. Define $Z=X+Y$, and let $\left(\mathcal{F}_{t}\right)$ be the natural filtration of $Z$. Let $\left(\mathcal{F}_{t}^{X}\right)$ and $\left(\mathcal{F}_{t}^{Y}\right)$ be the filtration generated by the processes $X$ and $Y$, respectively.

Fix $t \in[0, T], f \in C_{0}[t, T]$, and $\varepsilon>0$. By the tower property of conditional expectations,

$$
\begin{aligned}
& \mathrm{P}\left(\sup _{u \in[t, T]}\left|Z_{u}-Z_{t}-f(u)\right|<\varepsilon \mid \mathcal{F}_{t}\right) \\
& \quad=\mathrm{E}\left(\mathrm{P}\left(\sup _{u \in[t, T]}\left|Z_{u}-Z_{t}-f(u)\right|<\varepsilon \mid \mathcal{F}_{t}^{X} \vee \mathcal{F}_{T}^{Y}\right) \mid \mathcal{F}_{t}\right) .
\end{aligned}
$$

By independence, the inner conditional probability equals $\Xi\left(\left(f(u)-\left(Y_{u}-Y_{t}\right)\right)_{u \in[t, T]}, t\right)$, where, for $g \in C_{0}[t, T]$,

$$
\Xi(g, t)=\mathrm{P}\left(\sup _{u \in[t, T]}\left|X_{u}-X_{t}-g(u)\right|<\varepsilon \mid \mathcal{F}_{t}^{X}\right) .
$$

Hence, since $X$ has CFS, the inner probability is strictly positive almost surely. By the strict 
positivity of the conditional expectation operator, it also follows that

$$
\mathrm{P}\left(\sup _{u \in[t, T]}\left|Z_{u}-Z_{t}-f(u)\right|<\varepsilon \mid \mathcal{F}_{t}\right)>0
$$

almost surely.

\subsection{Proof of Theorem 2.1}

We begin with the proof of part (i) and Corollary 2.1. First observe that $X$ can be written as the sum of two independent continuous Gaussian si-processes, one with spectral density $f$ and one with a singular spectral measure $\mu_{s}$. In view of Lemma 3.2 it suffices to show that the process with spectral density $f$ has CFS. Therefore, we proceed without loss of generality under the assumption that $\mu$ is absolutely continuous, i.e. that $\mu_{s}=0$.

The condition on $f$ implies that $f(\lambda)>0$ for almost every $\lambda$ outside $\left[-\lambda_{0}, \lambda_{0}\right]$. Since changing $f$ on a Lebesgue null set does not change the law of the si-process, we may assume that $f$ is strictly positive outside $\left[-\lambda_{0}, \lambda_{0}\right]$. Now define a new spectral density $g: \mathbb{R} \rightarrow \mathbb{R}$ by

$$
g(\lambda)= \begin{cases}f(\lambda), & |\lambda|>\lambda_{0}, \\ \lambda^{2}, & |\lambda| \leq \lambda_{0}\end{cases}
$$

Let $Y$ be a Gaussian si-process with spectral density $g$. By construction, it holds that

$$
\int_{\mathbb{R}} \frac{\log g(\lambda)}{1+\lambda^{2}} \mathrm{~d} \lambda>-\infty .
$$

Since the spectral densities $f$ and $g$ are equal outside a bounded set, Theorem A.1 in Appendix A then implies that the laws of $\left(X_{t}: t \in[0, T]\right)$ and $\left(Y_{t}: t \in[0, T]\right)$ are equivalent. Hence, by Lemma 3.1, it suffices to prove that the process $\left(Y_{t}: t \in[0, T]\right)$ with spectral density $g$ has CFS.

It follows from the assumption on $f$ and the construction of $g$ that

$$
\int_{\mathbb{R}} \frac{g(\lambda)}{\lambda^{2}} \mathrm{~d} \lambda<\infty
$$

and

$$
\int_{\mathbb{R}} \frac{\log g(\lambda) / \lambda^{2}}{1+\lambda^{2}} \mathrm{~d} \lambda>-\infty
$$

Indeed, note that since $f$ is a density, it has a finite integral outside $\left[\lambda_{0}, \lambda_{0}\right]$, and $g(\lambda)=\lambda^{2}$ inside $\left[\lambda_{0}, \lambda_{0}\right]$. Hence, by Theorem A.3 in Appendix A, we have the representation $g(\lambda) / \lambda^{2}=|\psi(\lambda)|^{2}$ for a Hardy function $\psi \in \mathbb{H}^{2+}$ satisfying $\overline{\psi(\lambda)}=\psi(-\lambda)$; cf. Dym and McKean (1976, p. 38). By the Paley-Wiener theorem for Hardy functions (see Theorem A.2 in Appendix A) we have $\psi=\hat{K}$ for some real valued $K \in L^{2}[0, \infty)$. We conclude that $g(\lambda)=\lambda^{2}|\hat{K}(\lambda)|^{2}$. But this implies that the process $Y$ with spectral density $g$ can be realized as the moving average

$$
Y_{t}=2 \pi \int_{-\infty}^{t}(K(t-s)-K(-s)) \mathrm{d} B_{s},
$$

where $B$ is a Brownian motion. Indeed, it follows from the Parseval relation that

$$
\int_{\mathbb{R}} K(t-s) \mathrm{e}^{\mathrm{i} \lambda s} \mathrm{~d} s=\mathrm{e}^{\mathrm{i} \lambda t} \overline{\hat{K}(\lambda)} .
$$


So, for the moving average (3.1), we have

$$
\mathrm{E} Y_{S} Y_{t}=\int_{\mathbb{R}}\left(\mathrm{e}^{\mathrm{i} \lambda s}-1\right)\left(\mathrm{e}^{-\mathrm{i} \lambda t}-1\right)|\hat{K}(\lambda)|^{2} \mathrm{~d} \lambda,
$$

which means that (3.1) indeed has spectral density $\lambda^{2}|\hat{K}(\lambda)|^{2}$.

Finally, by Theorem 1.1 of Cherny (2008), the moving average process ( $\left.Y_{t}: t \in[0, T]\right)$ has CFS, which completes the proof of part (i) and Corollary 2.1 .

The idea of the proof of statement (ii) is the following heuristic. A process with exponentially decaying spectral density has very smooth, even analytic, paths. Therefore, it is enough to observe the realized path on any interval $[0, \varepsilon]$ to have complete knowledge of the whole realized path.

More rigorously, we note that, by the condition of the theorem and the fact that $\mu$ is a spectral measure and, hence, $\int\left(1+\lambda^{2}\right)^{-1} \mu(\mathrm{d} \lambda)<\infty$, we have

$$
\int_{\mathbb{R}} \mathrm{e}^{a|\lambda|} \mu(\mathrm{d} \lambda)<\infty
$$

It follows that the polynomials lie dense in the space $L^{2}(\mu)$ of functions on the line that are square integrable with respect to $\mu$; cf. e.g. Parthasarathy (2005, Proposition 6.4.1). Now let $(\Omega, \mathcal{F}, \mathrm{P})$ be the underlying probability space on which the process $X$ is defined, and let $\left(\mathscr{F}_{t}\right)$ be its natural filtration. Then the spectral representation (1.1) defines a Hilbert space isometry between the linear span $\mathscr{H}$ of the collection of random variables $\left\{X_{t}: t \geq 0\right\}$ in $L^{2}(\Omega, \mathcal{F}, \mathrm{P})$ and the linear span $\mathcal{L}$ of the collection of functions $\left\{e_{t}: t \geq 0\right\}$ in $L^{2}(\mu)$, where

$$
e_{t}(\lambda)=\frac{\mathrm{e}^{\mathrm{i} \lambda t}-1}{\mathrm{i} \lambda} .
$$

This isometry $S: \mathscr{H} \rightarrow \mathcal{L}$ is called the spectral isometry and is determined by the fact that $S\left(X_{t}\right)=e_{t}$ for every $t \geq 0$. Relation (3.2) implies that $\mu$ has finite moments of all orders. The process $X$ is therefore infinitely often differentiable in the mean-square sense; cf. Doob (1953, pp. 535-537 and 558). Denoting the $k$ th derivative by $X^{(k)}$ we have $X_{t}^{(k)} \in \mathcal{H}$ for all $k \in \mathbb{N}$ and $t \geq 0$. By repeatedly differentiating (1.1) we see that, for the image of $X_{t}^{(k)}$ under the spectral isometry, it holds that

$$
\left(S\left(X_{t}^{(k)}\right)\right)(\lambda)=(\mathrm{i} \lambda)^{k-1} \mathrm{e}^{\mathrm{i} \lambda t}
$$

for $k \in \mathbb{N}$ and $t \geq 0$. Since the polynomials are dense in $\mathcal{L}$, it follows that the random variables $\left\{X_{0}^{(k)}: k \in \mathbb{N}\right\}$ span the whole space $\mathcal{H}$. In particular, every variable $X_{t}$ is an $L^{2}$-limit of linear combinations of random variables of the form $X_{0}^{(k)}$. Since these variables are $\mathcal{F}_{s}$-measurable for every $s>0$, this shows that $\mathcal{F}_{s}=\mathscr{F}_{t}$ for all $0<s<t$. In other words, the process $X$ is completely determined by what happens immediately after time 0 . It follows that, for $t \in(0, T)$, the support of $\left(X_{u}: u \in[t, T]\right)$ given $\mathcal{F}_{t}$ equals the support of $\left(X_{u}: u \in[t, T]\right)$ given $\mathcal{F}_{T}$. The latter is obviously almost surely degenerate, which implies that the process does not have CFS. This completes the proof of part (ii).

\section{Appendix A}

For the convenience of the reader, we collect in this appendix some known facts that we used in the proof of our main theorem about the equivalence of Gaussian si-processes and about Hardy functions. 


\section{A.1. Equivalence of Gaussian si-processes}

Let $X$ be a continuous Gaussian si-process with spectral measure $\mu$ (as defined in the introduction), defined on a probability space $(\Omega, \mathcal{F}, \mathrm{P})$. For every $t>0$, let $\mathscr{H}_{t}$ be the closure in $L^{2}(\Omega, \mathcal{F}, \mathrm{P})$ of the linear span of the random variables $\left\{X_{s}: 0 \leq s \leq t\right\}$ and let $\mathcal{L}_{t}$ be the closure in $L^{2}(\mu)$ of the linear span of the collection of functions $\left\{e_{s}: s \in[0, t]\right\}$, where

$$
e_{t}(\lambda)=\frac{\mathrm{e}^{\mathrm{i} \lambda t}-1}{\mathrm{i} \lambda}
$$

The spectral representation (1.1) implies that we have a Hilbert space isometry between $\mathscr{H}_{T}$ and $\mathcal{L}_{T}$, determined by the identification $X_{t} \leftrightarrow e_{t}$.

Krein's alternative, a central result in the theory of stationary and si-processes, implies that, for every $T>0$, the space $\mathcal{L}_{T}$ is either a reproducing kernel Hilbert space (RKHS) of entire functions or $\mathcal{L}_{T}=L^{2}(\mu)$; cf. Dym and McKean (1976, Section 6.4). We use the following sufficient condition for the former case. See, e.g. Dym and McKean (1976, Exercise 5, p. 247).

Lemma A.1. Let $f$ be the Lebesgue density of the absolutely continuous part of the spectral measure $\mu$ of the Gaussian si-process $X$. If

$$
\int_{\mathbb{R}} \frac{\log f(\lambda)}{1+\lambda^{2}} \mathrm{~d} \lambda>-\infty
$$

then, for every $T>0, \mathcal{L}_{T}$ is an RKHS of entire functions.

Now suppose that $\mu$ is absolutely continuous, and let $f$ be the spectral density of $X$, i.e. the Lebesgue density of $\mu$. Let $Y$ be a second Gaussian si-process with absolutely continuous spectral measure, with spectral density $g$. The following theorem essentially states that, for the equivalence of si-processes, we have to consider only the spectral measure outside a bounded set. For a proof, see van Zanten (2007, Theorem 5.1).

Theorem A.1. Suppose that the spectral density $f$ of $X$ satisfies (A.1) and that $f$ is strictly positive outside a bounded set. Then if $f=g$ outside a bounded set, the laws of $\left(X_{t}: t \in[0, T]\right)$ and $\left(Y_{t}: t \in[0, T]\right)$ are equivalent for every $T>0$.

\section{A.2. Hardy functions}

Hardy functions play an important role in the theory of stationary and si-processes. We recall a few facts needed in the proof of Theorem 2.1.

Definition A.1. A function $h$ that is analytic in the complex upper half-plane $\mathbb{C}^{+}=\{a+\mathrm{i} b: a \in$ $\mathbb{R}, b>0\}$ is said to belong to the Hardy class $\mathbb{H}^{2+}$ if

$$
\|h\|_{2+}=\sup _{b>0} \sqrt{\int_{\mathbb{R}}|h(a+\mathrm{i} b)|^{2} \mathrm{~d} a}<\infty .
$$

The Paley-Wiener theorem for Hardy functions asserts that the Hardy functions are exactly the Fourier transforms of functions that vanish on the negative half-line. See, e.g. Section 2.3 of Dym and McKean (1976), or Theorem 19.2 of Rudin (1987).

Theorem A.2. If $h \in \mathbb{H}^{2+}$ then there exists a function $f \in L^{2}[0, \infty)$ such that

$$
h(z)=\int_{0}^{\infty} \mathrm{e}^{\mathrm{i} z x} f(x) \mathrm{d} x
$$

for all $z \in \mathbb{C}^{+}$. Moreover, $\|f\|_{L^{2}[0, \infty)}^{2}=2 \pi\|h\|_{2+}^{2}$. 
For a Hardy function $h \in \mathbb{H}^{2+}$ and $b>0$, we define $h_{b} \in L^{2}(\mathbb{R})$ by $h_{b}(a)=h(a+\mathrm{i} b)$. The preceding theorem implies that, for a function $f \in L^{2}[0, \infty)$, the function $h_{b}$ converges in $L^{2}(\mathbb{R})$ to a function $h_{0+}$ as $b \downarrow 0$, given by

$$
h_{0+}(a)=\int_{0}^{\infty} \mathrm{e}^{\mathrm{i} a x} f(x) \mathrm{d} x .
$$

The function $h_{0+}$ in fact completely determines $h$ and satisfies the bound

$$
\int_{\mathbb{R}} \frac{\log \left|h_{0+}(\lambda)\right|^{2}}{1+\lambda^{2}} \mathrm{~d} \lambda>-\infty ;
$$

cf. Section 2.6 of Dym and McKean (1976).

The following result gives a converse statement. See, e.g. Dym and McKean (1976, p. 38).

Theorem A.3. Let $f$ be a nonnegative, integrable function on $\mathbb{R}$. If

$$
\int_{\mathbb{R}} \frac{\log f(\lambda)}{1+\lambda^{2}} \mathrm{~d} \lambda>-\infty
$$

then $f=\left|h_{0+}\right|^{2}$ for some Hardy function $h \in \mathbb{H}^{2+}$. If $f$ is symmetric then $\overline{h_{0+}(\lambda)}=h_{0+}(-\lambda)$ for all $\lambda \in \mathbb{R}$.

\section{References}

Bender, C., Sottinen, T. and Valkeila, E. (2008). Pricing by hedging and no-arbitrage beyond semimartingales. Finance Stoch. 12, 441-468.

Cherny, A. (2008). Brownian moving averages have conditional full support. Ann. Appl. Prob. 18, 1825-1830.

Delbaen, F. and Schachermayer, W. (2006). The Mathematics of Arbitrage. Springer, Berlin.

Doob, J. L. (1953). Stochastic Processes. John Wiley, New York.

Dym, H. and McKean, H. P. (1976). Gaussian Processes, Function Theory, and the Inverse Spectral Problem. Academic Press, New York.

Guasoni, P., RÁsonyi, M. AND Schachermayer, W. (2008a). Consistent price systems and face-lifting pricing under transaction costs. Ann. Appl. Prob. 18, 491-520.

GuAsoni, P., RÁSONYi, M. AND SCHACHERMAYER, W. (2008b). The fundamental theorem of asset pricing for continuous processes under small transaction costs. Ann. Finance 6, 157-191.

Jarrow, R. A., Protter, P. And SAYit, H. (2009). No arbitrage without semimartingales. Ann. Appl. Prob. 19, $596-616$.

Parthasarathy, K. R. (2005). Introduction to Probability and Measure. Hindustan Book Agency, New Delhi.

Rudin, W. (1987). Real and Complex Analysis, 3rd edn. McGraw-Hill, New York.

Samorodnitsky, G. And TaquU, M. S. (1994). Stable Non-Gaussian Random Processes. Chapman and Hall, New York.

Van Zanten, H. (2007). When is a linear combination of independent fBm's equivalent to a single fBm? Stoch. Process. Appl. 117, 57-70. 